\title{
What powers the radio-loud narrow-line Seyfert 1 galaxy $\mathrm{RX} \mathrm{J} 2314.9+2243 ?$
}

\section{A view onto its central engine from radio to X-rays ${ }^{\star}$}

\author{
S. Komossa ${ }^{1}$, Dawei Xu ${ }^{2}$, L. Fuhrmann ${ }^{1}$, D. Grupe ${ }^{3,4}$, S. Yao $^{2}$, Z. Fan ${ }^{2}$, I. Myserlis ${ }^{1}$, E. Angelakis ${ }^{1}$, V. Karamanavis ${ }^{1}$, \\ W. Yuan ${ }^{2}$, and J. A. Zensus ${ }^{1}$
}

${ }^{1}$ Max-Planck-Institut für Radioastronomie, Auf dem Hügel 69, 53121 Bonn, Germany
e-mail: skomossa@mpifr.de
2 National Astronomical Observatories, Chinese Academy of Sciences, Beijing 100012, PR China
3 Space Science Center, Morehead State University, 235 Martindale Dr., Morehead, KY 40351, USA
4 Swift Mission Operation Center, 2582 Gateway Dr., State College, PA 16801, USA

Received 15 August 2014 / Accepted 24 October 2014

\begin{abstract}
Aims. Radio-loud narrow-line type 1 galaxies provide us with a fresh look at the blazar phenomenon, the causes of radio loudness, and the physics of jet formation. We present a multi-wavelength study of the radio-loud narrow-line type 1 quasar RX J2314.9+2243, which exhibits some remarkable multi-wavelength properties. It is among the few radio-loud narrow-line type 1 galaxies, with a tentative $\gamma$-ray detection, is luminous in the infrared, and shows an exceptionally broad and blueshifted [OIII] $\lambda 5007$ emission-line component.

Methods. In order to understand the nature of this source, we have obtained optical, UV, X-ray, and radio observations of RX J2314.9+2243.

Results. Its spectral energy distribution (SED) shows a broad hump extending between the IR and far-UV, a steep radio spectrum and flat X-ray spectrum. Its IR to far-UV SED is consistent with a scenario, in which synchrotron emission from a jet dominates the broadband emission, even though an absorption scenario cannot yet be fully excluded. The high blueshift of its very broad [OIII] component, $1260 \mathrm{~km} \mathrm{~s}^{-1}$, is consistent with a face-on view, with the jet (and outflow) pointing towards us. RX J2314.9+2243 likely represents an extreme case of AGN induced feedback in the local universe.
\end{abstract}

Key words. galaxies: active - galaxies: evolution - galaxies: Seyfert - galaxies: individual: RX J2314.9+2243

\section{Introduction}

About $15-20 \%$ of all active galactic nuclei (AGN) are radioloud (Urry \& Padovani 1995), for reasons that are not yet well understood. It has been suggested that the influence of spin, magnetic fields, and/or host galaxy properties play an important role (e.g., Wilson \& Colbert 1995; Capetti \& Balmaverde 2006; Tchekhovskoy et al. 2010; Sikora \& Begelman 2013a). In recent years, a new sub-class of radio-loud AGN has emerged, providing us with new insights into this old problem. These are the narrow-line Seyfert 1 (NLS1) galaxies and their higherluminosity counterparts, the narrow-line type 1 quasars. These galaxies are characterized by their optical emission-line spectra, which show broad Balmer lines that are narrower than the rest of the Seyfert 1 population; $F W M H(\mathrm{H} \beta) \leq 2000 \mathrm{~km} \mathrm{~s}^{-1}$ (e.g., Osterbrock \& Pogge 1985; Goodrich 1989; Véron-Cetty et al. 2001). NLS1 galaxies exhibit many extreme properties among AGN, including, on average, super-strong FeII emission, rapid X-ray variability, near-Eddington accretion rates, strong star formation, and rapidly growing low-mass black holes

^ The optical spectrum (FITS format) is only available at the CDS via anonymous ftp to cdsarc.u-strasbg. fr (130.79.128.5) or via http://cdsarc.u-strasbg.fr/viz-bin/qcat?]/A+A/574/A121 (e.g., Boroson \& Green 1992; Boller et al. 1996; Leighly 1999; Mathur 2000; Grupe 2004; Zhou et al. 2006; Sani et al. 2010; $\mathrm{Xu}$ et al. 2012; review by Komossa 2008).

A dedicated search for strong radio emission from 128 NLS1 galaxies (Komossa et al. 2006; hereafter K06) has shown that only $7 \%$ of all NLS1 galaxies are radio-loud $(R>$ $10)^{1}$, while only $2.5 \%$ exceed $R>100$. These fractions are much lower than among broad-line Seyfert 1 (BLS1) galaxies $(20 \%$ and $13 \%$, respectively; K06). Early studies have shown that the radio-loud NLS1 galaxies come in two flavors. While some of them are compact steep-spectrum radio sources, the others have flat or inverted radio spectra, are variable, and several of them show strong signs of relativistic beaming (Zhou et al. 2003; K06; Yuan et al. 2008), properties reminiscent of blazars.

Host galaxy morphologies of the radio-loud narrow-line type 1 quasars and Seyferts are generally not yet known. An exception is the remarkable NLS1 galaxy $1 \mathrm{H} 0323+342$, which is hosted by a one-armed spiral or ring galaxy (Zhou et al. 2007; Anton et al. 2008). It remains to be seen whether this is the exception or the rule. Radio-loud NLS1 galaxies are typically very compact radio sources. High-resolution radio imaging

\footnotetext{
1 Kellerman's radio index $R$ is defined as the ratio of the $6 \mathrm{~cm}$ radio flux density to the optical flux density at 4400 A (Kellerman et al. 1989).
} 
Table 1. Journal of observations.

\begin{tabular}{|c|c|c|c|}
\hline Waveband & Instrument & Date of observation & Exposure time \\
\hline \multirow[t]{2}{*}{ Optical } & Xinglong $2.2 \mathrm{~m}, 300$ line $/ \mathrm{mm}$ grating & 1998 Sep. 21 & $2100 \mathrm{~s}$ \\
\hline & Xinglong $2.2 \mathrm{~m}, 600$ line $/ \mathrm{mm}$ grating & 2005 Nov. 25 & $3600 \mathrm{~s}$ \\
\hline \multirow[t]{2}{*}{ Optical } & Xinglong $80 \mathrm{~cm}, R$ band & 2013 Nov. $2-4$, Dec. $28-30$ & $200 \mathrm{~s}$ \\
\hline & Xinglong $80 \mathrm{~cm}, B$ band & 2013 Nov. 2-4, Dec. $28-30$ & $400-600 \mathrm{~s}$ \\
\hline \multirow[t]{2}{*}{$V$} & Swift UVOT & 2007 May 12 & $165 \mathrm{~s}$ \\
\hline & & 2007 May 20 & $1188 \mathrm{~s}$ \\
\hline \multirow[t]{2}{*}{$B$} & Swift UVOT & 2007 May 12 & $166 \mathrm{~s}$ \\
\hline & & 2007 May 20 & $1187 \mathrm{~s}$ \\
\hline \multirow[t]{2}{*}{$U$} & Swift UVOT & 2007 May 12 & $165 \mathrm{~s}$ \\
\hline & & 2007 May 20 & $1187 \mathrm{~s}$ \\
\hline \multirow[t]{2}{*}{$U V W 1$} & Swift UVOT & 2007 May 12 & $332 \mathrm{~s}$ \\
\hline & & 2007 May 20 & $2378 \mathrm{~s}$ \\
\hline \multirow[t]{2}{*}{$U V M 2$} & Swift UVOT & 2007 May 12 & $387 \mathrm{~s}$ \\
\hline & & 2007 May 20 & $2703 \mathrm{~s}$ \\
\hline \multirow[t]{2}{*}{$U V W 2$} & Swift UVOT & 2007 May 12 & $665 \mathrm{~s}$ \\
\hline & & 2007 May 20 & $4761 \mathrm{~s}$ \\
\hline \multirow[t]{2}{*}{ X-rays } & Swift XRT & 2007 May 12 & $1960 \mathrm{~s}$ \\
\hline & & 2007 May 20 & $13860 \mathrm{~s}$ \\
\hline \multirow[t]{4}{*}{ Radio } & Effelsberg $100 \mathrm{~m}$ & 2013 Feb. 03 & $2280 \mathrm{~s}$ \\
\hline & & 2013 Feb. 09 & $3300 \mathrm{~s}$ \\
\hline & & 2013 July 07 & $2460 \mathrm{~s}$ \\
\hline & & 2013 July 23 & $2280 \mathrm{~s}$ \\
\hline
\end{tabular}

only exists for a few of them (e.g., Dallacasa et al. 1998; Doi et al. 2007, 2011, 2012; Gu \& Chen 2010; Giroletti et al. 2011; Wajima et al. 2014).

Recently, several of the radio-loud NLS1 galaxies have been detected at $\gamma$-rays for the first time (e.g., Abdo et al. 2009a,b; Foschini 2011; Foschini et al. 2011, 2014; Calderone et al. 2011; D'Ammando et al. 2012, 2013) with the Fermi observatory. Some show repeated, rapid flaring, interpreted as emission from relativistic jets. A marginal $\mathrm{TeV}$ detection was reported for $1 \mathrm{H} 0323+342$ (Falcone et al. 2004). Multifrequency radio monitoring campaigns of several of the $\gamma$-ray detected NLS1 galaxies reveal flux density and spectral index variability including strong spectral evolution (e.g., Fuhrmann et al. 2011; Foschini et al. 2012; D’Ammando et al. 2014; Angelakis et al. 2014).

We are just beginning to understand this intriguing population of sources. In particular, some of the main properties of classical NLS1 galaxies, including their high Eddington accretion rates and low black hole masses, are unlike those of most classical blazars. Either radio-loud beamed NLS1 galaxies extend the classical blazar phenomenon towards lower SMBH (supermassive black hole) and host masses, or else they represent a new class of sources with relativistic jets. In any case, they provide us with important new insights into the phenomenon of radio-loudness, and the formation of radio jets under extreme conditions.

Here, we report on dedicated optical, UV, X-ray and radio observations of RX J2314.9+2243 $\left(\alpha_{2000}=23^{\mathrm{h}} 14^{\mathrm{s}} 55.7^{\mathrm{s}}\right.$; $\left.\delta_{2000}=+22^{\circ} 43^{\prime} 25^{\prime \prime} ; z=0.1692\right)$. RX J2314.9+2243 is one of only about a dozen NLS1 galaxies detected at $\gamma$-rays (Foschini, priv. comm. ${ }^{2}$; Berton et al., in prep.). It was identified as a quasar with an absolute magnitude $M_{B}=-23.6$ (Wei et al. 1998). RX J2314.9+2243 was found to be mildly radio-loud, with a radio index $R=10-20$ (K06). It is detected by IRAS and luminous in the infrared.

While it is common to call the whole source population of narrow-line type $1 \mathrm{AGN}$ as narrow-line Seyfert 1 galaxies,

\footnotetext{
2 http://www.brera.inaf.it/utenti/foschini/gNLS1/ catalog.html
}

we note that a fraction of them is actually very luminous, and so they would formally be called quasars; i.e., narrow-line type 1 quasars. Even though RX J2314.9+2243 is a quasar, throughout this paper we follow common habit and refer to it as NLS1 galaxy from now on.

This paper is structured as follows: In Sects. 2-5 we present the optical, UV, X-ray, and radio data, respectively, including the first simultaneous optical-X-ray measurements and the first simultaneous multifrequency radio measurements of this galaxy. Archival data are added in Sect. 6 for obtaining the broadband spectral energy distribution (SED). A comment on black hole mass and Eddington ratio is given in Sect. 7. The discussion follows in Sects. 8, and 9 provides a summary and conclusions. Throughout this paper, we use a cosmology (Wright 2006) with $H_{0}=75 \mathrm{~km} \mathrm{~s}^{-1} \mathrm{Mpc}^{-1}, \Omega_{\mathrm{M}}=0.3$, and $\Omega_{\Lambda}=0.7$.

\section{Optical spectroscopy}

\subsection{Optical spectroscopic observations and data reduction}

We took spectra of RX J2314.9+2243 with the Opto-Mechanics Research (OMR) spectrograph attached to the Xinglong $2.16 \mathrm{~m}$ telescope twice. The low-resolution spectrum was taken on 1998 September 21 with a slit width of $2.3^{\prime \prime}$, using a Tektronix $1024 \times$ 1024 CCD as detector. A 300 line $\mathrm{mm}^{-1}\left(200 \AA \mathrm{mm}^{-1}\right)$ grating was employed to obtain the broadband optical spectrum. The exposure time was $2100 \mathrm{~s}$, at an airmass of 1.089 .

Motivated by the exceptional structure of its [O III] profile (K06), we obtained a moderate-resolution spectrum of RX J2314.9+2243 on 2005 November 25. A Tektronix $1300 \times$ $1024 \mathrm{CCD}$ and a 600 line $\mathrm{mm}^{-1}\left(100 \AA \mathrm{mm}^{-1}\right)$ grating was used. The observation was carried out through a slit width of $2.3^{\prime \prime}$. The spectral resolution as measured from the comparison spectra was $11 \AA$ (for the 300 line $\mathrm{mm}^{-1}$ grating) and $5.5 \AA$ (600 line $\mathrm{mm}^{-1}$ grating) at FWHM, respectively. The exposure time was $3600 \mathrm{~s}$, and the airmass 1.088. A log of the observations is given in Table 1.

Data reduction was carried out following the standard procedures using the package IRAF. The CCD reduction included 

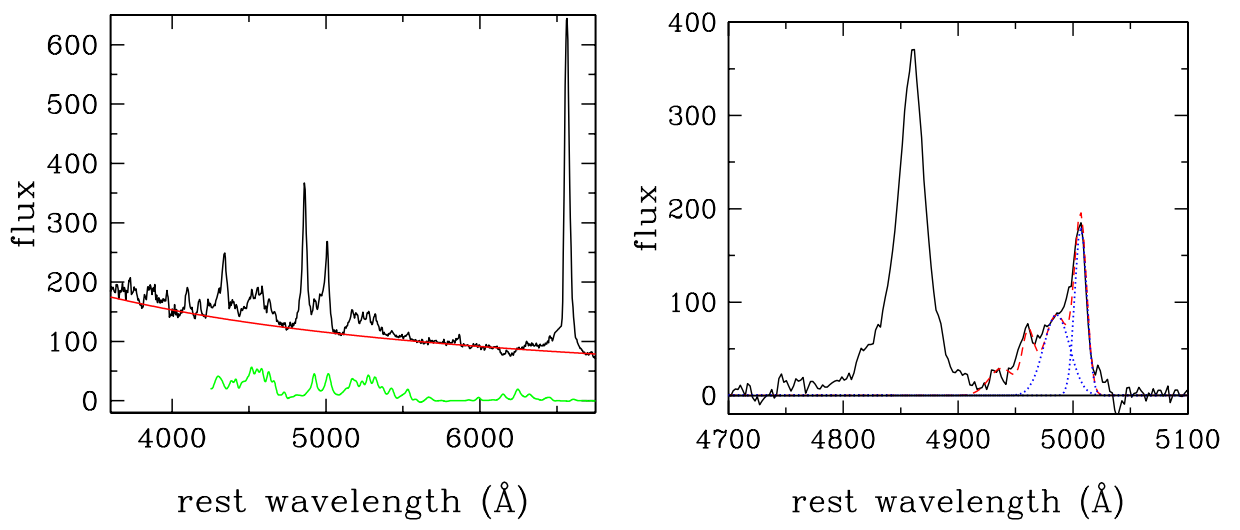

Fig. 1. Left: low-resolution optical spectrum of RX J2314.9+2243, and the best-fit powerlaw continuum and Fe II complexes. Right: zoom onto the $\mathrm{H} \beta-[\mathrm{OIII}]$ regime (moderate resolution). The dashed line shows our double-Gaussian fit to [OIII]4959,5007. The dotted lines represent the narrow and broad components of [OIII]5007. The flux density $f_{\lambda}$ is given in units of $10^{-17} \mathrm{erg} \mathrm{s}^{-1} \mathrm{~cm}^{-2} \AA^{-1}$. (A color version of this figure is available in the online journal.)

Table 2. Emission-line properties of RX J2314.9+2243.

\begin{tabular}{llrrl}
\hline \hline Line identification & Model $^{a}$ & $F W H M^{b}$ & Line ratio & Comments \\
\hline$[\mathrm{O}$ III $] \lambda 5007_{\text {total }}$ & $\mathrm{g}+\mathrm{g}$ & $\ldots$. & 0.37 & \\
{$[\mathrm{O} \mathrm{III}]_{\text {narrow }}$} & $\mathrm{g}+\mathrm{g}$ & 620 & 0.18 & \\
{$[\mathrm{O} \text { III }]_{\text {broad }}$} & $\mathrm{g}+\mathrm{g}$ & 1560 & 0.19 & blueshifted from the narrow component by $1260 \mathrm{~km} \mathrm{~s}^{-1}$ \\
$\mathrm{H} \beta_{\text {total }}$ & $\mathrm{g}$ & 1670 & 0.87 & \\
& $\mathrm{~g}$ & 1580 & 1.22 & \\
$\mathrm{H} \beta_{\text {total }}$ & $\mathrm{d}$ & 1630 & 1.03 & \\
$\mathrm{H} \beta_{\text {narrow }}$ & $\mathrm{g}+\mathrm{g}$ & $\ldots$. & 1.0 & \\
$\mathrm{H} \beta_{\text {broad }}$ & $\mathrm{g}+\mathrm{g}$ & 620 & 0.15 & $F H H M\left(\mathrm{H} \beta_{\text {narrow }}\right)$ fixed to $F W H M\left([\mathrm{O} \mathrm{III}]_{\text {narrow }}\right)$ \\
$\mathrm{H} \beta_{\text {total }}$ & $\mathrm{g}+\mathrm{g}$ & 2680 & 0.85 & \\
$\mathrm{H} \beta_{\text {narrow }}$ & $\mathrm{g}+\mathrm{l}$ & $\ldots$. & 1.22 & \\
$\mathrm{H} \beta_{\text {broad }}$ & $\mathrm{g}+1$ & 620 & 0.02 & Gaussian profile with $F H H M\left(\mathrm{H} \beta_{\text {narrow }}\right)$ fixed to $F W H M\left([\mathrm{O} \text { III }]_{\text {narrow }}\right)$ \\
$\mathrm{H} \alpha+[\mathrm{N} \mathrm{III}] \lambda \lambda 6548,6583$ & $\mathrm{~g}+1$ & 1620 & 1.20 & Lorentzian profile \\
$\mathrm{H} \gamma$ & $\mathrm{d}$ & 1550 & $<3.3^{\mathrm{c}}$ & \\
\hline
\end{tabular}

Notes. ${ }^{(a)}$ Models used to fit emission lines (g: Gaussian profile; 1: Lorentzian profile; d: direct integration over the observed profile; g+g: doubleGaussian profile; g+l: Gaussian-plus-Lorentzian profile). ${ }^{(b)}$ In units of $\mathrm{km} \mathrm{s}^{-1}$. Corrected for instrumental resolution. ${ }^{(c)} \mathrm{Measured}$ relative to $\mathrm{H} \beta$ in the low-resolution spectrum.

bias subtraction, flatfield correction and cosmic-ray removal. Wavelength calibration was performed using helium-neon-argon lamps taken at the beginning and end of each exposure. Flux calibration was accomplished with 2 to 3 exposures of KPNO standard stars (Massey \& Strobel 1988) per night.

A Galactic extinction correction was applied to the wavelength- and flux-calibrated spectra, with $E_{B-V}=0.195 \mathrm{mag}$ (Schlegel et al. 1998) and a standard $R=3.1$ extinction law. The final calibrated broadband spectrum, corrected for Galactic extinction, and a zoom on the $\mathrm{H} \beta-[\mathrm{OIII}]$ complex with higher resolution, are shown in Fig. 1.

\subsection{Spectral fits}

The spectrum of RX J2314.9+2243 shows an AGN dominated continuum and strong FeII emission on the blue and red side of the $\mathrm{H} \beta$-[OIII] complex. We used the IRAF SPECFIT package (Kriss 1994) for spectral analysis. We first fitted the spectrum with (1) a powerlaw continuum; and (2) broad optical FeII emission, using the FeII template of Boroson \& Green (1992). The FeII template was convolved according to the line width of $\mathrm{H} \beta$ and then scaled to match the line intensities. The continuum-subtracted and FeII-subtracted spectrum was then used to measure emission line properties. No additional host galaxy contribution was included in the fitting.

Emission lines were analyzed by fitting single Gaussian profiles and combinations of Gaussian and/or Lorentzian profiles.
Both profile shapes have been commonly employed to represent broad emission-line shapes of AGN (e.g., Evans 1988). Occasionally, Lorentzians have been preferred in describing the Balmer line profiles of NLS1 galaxies (e.g., Véron-Cetty et al. 2001). In this study, we use both types, and find that both are successful representations of the $\mathrm{H} \beta$ line profile.

In a first step, we focused on [OIII] $\lambda \lambda 4959,5007 \AA$, which show a remarkably strong second component which is highly blueshifted. In the fitting procedure, the width and redshift of the [OIII] doublet are required to be equal, and their flux ratio is fixed to the theoretical value. The double-Gaussian model describes each observed line profile well. As already briefly reported by K06, the broad blue wing, characterized by $F W H M=$ $1560 \mathrm{~km} \mathrm{~s}^{-1}$ is blueshifted by $1260 \mathrm{~km} \mathrm{~s}^{-1}$ from the narrow core of [OIII].

Next, $\mathrm{H} \beta$ was modeled. We tied the width of the narrow component of $\mathrm{H} \beta$ to that of [OIII] $\lambda 5007$ when fitting $\mathrm{H} \beta$ with double-Gaussian and Gaussian-plus-Lorentzian profiles. One Gaussian was used for the narrow component of the line. Its broad component was described by either a Gauss or Lorentz profile. We find that the line is similarly well fit by a double Gaussian and Gaussian-plus-Lorentzian profile. In the second case, the width of the broad component is $F W H M\left(\mathrm{H} \beta_{\text {broad }}\right)=1620 \mathrm{~km} \mathrm{~s}^{-1}$ while the Gauss approximation results in $F W H M\left(\mathrm{H} \beta_{\text {broad }}\right)=2680 \mathrm{~km} \mathrm{~s}^{-1}$. A direct measurement of the $\mathrm{H} \beta$ line width gives $F W H M\left(\mathrm{H} \beta_{\mathrm{d}}\right)=1630 \mathrm{~km} \mathrm{~s}^{-1}$. These results put RX J2314.9+2243 at the border between NLS1 and normal BLS1 galaxies. 
In order to assess the error in the line measurements, we have inspected the fluxes resulting from the different profile fits to $\mathrm{H} \beta$. Uncertainties arise from line deblending, and from the choice of Lorentzian or Gaussian profile to represent the broad emissionline component. This results in an uncertainty of $\sim 20 \%$ in line flux.

Next, we note that the $\mathrm{H} \alpha$ and $[\mathrm{NII}] \lambda \lambda 6548,6584$ complex is only covered by our low-resolution spectrum, in which $\mathrm{H} \alpha$ and the $[\mathrm{NII}]$ doublet are unresolved. For the sake of simplicity, we fitted $\mathrm{H} \alpha$ by a single Gauss profile with width and redshift fixed to that of $\mathrm{H} \beta$. Alternatively, the line flux was determined from a direct integration, without any assumptions on line shape. The same procedure was applied for $\mathrm{H} \beta$ in the low-resolution spectrum, in order to measure the Balmer decrement. Based on this method, we find a ratio $\mathrm{H} \alpha / \mathrm{H} \beta<3.3$, close to the recombination value (we note that the number is an upper limit, because of the extra contribution of $[\mathrm{NII}]$ to $\mathrm{H} \alpha$ ). Results are listed in Table 2. All quoted FWHMs were corrected for the instrumental resolution.

The broad FeII emission of RX J2314.9+2243 amounts to $\mathrm{R} 4570=1.2$, defined as the ratio of FeII $\lambda 4570$ over total $\mathrm{H} \beta$ emission, where the FeII emission complex is integrated between the rest wavelengths $4434 \AA$ and $4684 \AA$. The line ratio of [OIII]5007 over total $\mathrm{H} \beta$ emission is 0.4 . Therefore, RX J2314.9+2243 meets the classical optical classification criteria as a NLS1 galaxy. Its strong FeII emission relative to $\mathrm{H} \beta$ places it near the upper end of the FeII distribution among NLS1 galaxies (e.g., Grupe 2004; Xu et al. 2012).

Finally, we note that the spectrum of RX J2314.9+2243 also shows some additional FeII emission features, peaking around $\lambda_{\text {rest }}=4176 \AA$ and $4235 \AA$ (beyond the wavelength range covered by the FeII template of Boroson \& Green 1992). These features consist of a mix of allowed and forbidden FeII transitions, identified as narrow lines in the optical spectrum of $1 \mathrm{Zw}$ I by Véron-Cetty et al. (2004).

\section{Optical monitoring}

RX J2314.9+2243 is part of our optical monitoring campaign of radio-loud NLS1 galaxies, carried out at the Xinglong $80 \mathrm{~cm}$ TNT (Tsinghua-NAOC telescope). The telescope was equipped with a Princeton Instrument $1340 \times 1300$ thin back-illuminated CCD, which has a pixel size of $20 \mu \mathrm{m}$ and is cooled by liquid nitrogen. The field of view is $11.4^{\prime} \times 11.1^{\prime}$.

Observations were carried out in 2013 November 2-4 and 2013 December 28-30 with the standard Johnson-Cousin $R$ and $B$ filters, with typical exposure times of $200 \mathrm{~s}$ in $R$, and 400-600 $\mathrm{s}$ in $B$. The average seeing was around 2.0-2.3". Bias and flat-field corrections were first done. The photometry was performed within IDL with the task APER. Differential magnitudes were measured with respect to seven nearby stars in the same CCD frame which themselves did not show significant variability. The magnitudes of the comparison stars were taken from SDSS. Observed magnitudes of the stars were converted into the SDSS system by using the equations reported by Lupton $^{3}$. The lightcurve of RX J2314.9+2243 in the Johnson $R$ and $B$ band is shown in Fig. 2. We do not detect significant optical variability during the epoch of observations.

\footnotetext{
3 http://classic.sdss.org/dr5/algorithms/ sdssUBVRITransform.html\#Lupton2005
}

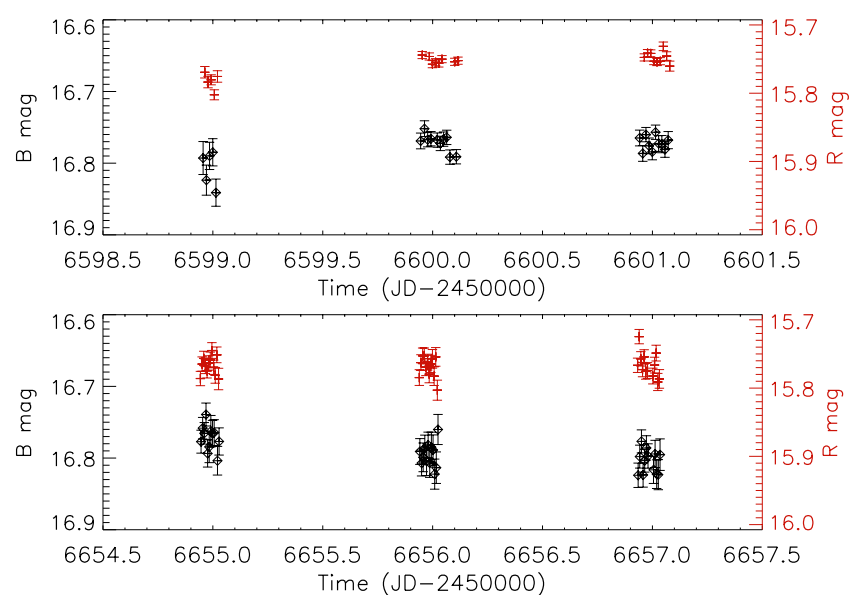

Fig. 2. Optical lightcurve of RX J2314.9+2243 in the $B$ (black) and $R$ (red) band. (A color version of this figure is available in the online journal.)

\section{Swift observations}

We have observed RX J2314.9+2243 twice with Swift, as part of our program to measure simultaneous optical-X-ray SEDs of radio-quiet (e.g., Grupe et al. 2010) and radio-loud NLS1 galaxies. RX J2314.9+2243 was observed on 2007 May 12 and 2007 May 20, with exposure times of 2.0 and $13.9 \mathrm{ks}$, respectively (target ID: 36553; Table 1).

Here, we present the UV and optical magnitude measurements obtained with the UV-optical telescope (UVOT; Roming et al. 2005), and X-ray data collected with the X-ray telescope (XRT; Burrows et al. 2005), sensitive to the 0.3-10 keV band. Since the UVOT magnitudes were constant within the errors at both dates of observation, and the X-ray countrate was lower during the first, shorter observation, we have performed the final measurements and spectral fits only on the data of the second observation.

\subsection{UVOT photometry}

In order to measure the optical-UV magnitudes, source counts in all 6 UVOT filters were first selected in a circular area with radius $5^{\prime \prime}$. The background was determined in a nearby, sourcefree region of radius $20^{\prime \prime}$. The task uvotsource, based on the most recent UVOT calibration (see Poole et al. 2008; Breeveld et al. 2010), was then used to measure fluxes and UVOT magnitudes. The UVOT data were corrected for Galactic reddening of $E_{B-V}=0.195$ (Schlegel et al. 1998). In each filter, the correction factor was derived according to Eq. (2) of Roming et al. (2009), based on the standard reddening correction curves by Cardelli et al. (1989). The UVOT magnitudes of RX J2314.9+2243 are given in Table 3. Based on a powerlaw fit to the UVOT data, we obtained an optical-UV spectral slope $\alpha_{\mathrm{UV}}=1.63\left(f \propto v^{\alpha}\right)$.

\subsection{X-ray observations}

RX J2314.9+2243 was detected in X-rays with XRT countrates of $(4.6 \pm 0.5) \times 10^{-2}$ counts $\mathrm{s}^{-1}$ and $(7.2 \pm 0.2) \times 10^{-2}$ counts s$^{-1}$, respectively, during the first and second observation.

The Swift XRT was operated in photon counting mode (Hill et al. 2004). Data reduction was carried out with the task $x r t$ pipeline version 0.12.6., which is included in the HEASOFT package 6.12. Source counts were selected in a circular region 
Table 3. Results of the Swift optical and UV photometry from 2007 May 20, corrected for Galactic extinction.

\begin{tabular}{lcc}
\hline \hline Filter & Magnitude & Flux $^{a}$ \\
\hline$V$ & $15.28 \pm 0.03$ & $1.59 \pm 0.05$ \\
$B$ & $15.69 \pm 0.02$ & $1.50 \pm 0.05$ \\
$U$ & $14.71 \pm 0.03$ & $1.61 \pm 0.07$ \\
$U V W 1$ & $14.99 \pm 0.03$ & $1.05 \pm 0.06$ \\
$U V M 2$ & $14.88 \pm 0.03$ & $1.14 \pm 0.04$ \\
$U V W 2$ & $15.34 \pm 0.03$ & $0.75 \pm 0.03$ \\
\hline
\end{tabular}

Notes. The results for 2007 May 12 are consistent within the errors. (a) In units of $10^{-11} \mathrm{erg} \mathrm{cm}^{-2} \mathrm{~s}^{-1}$.

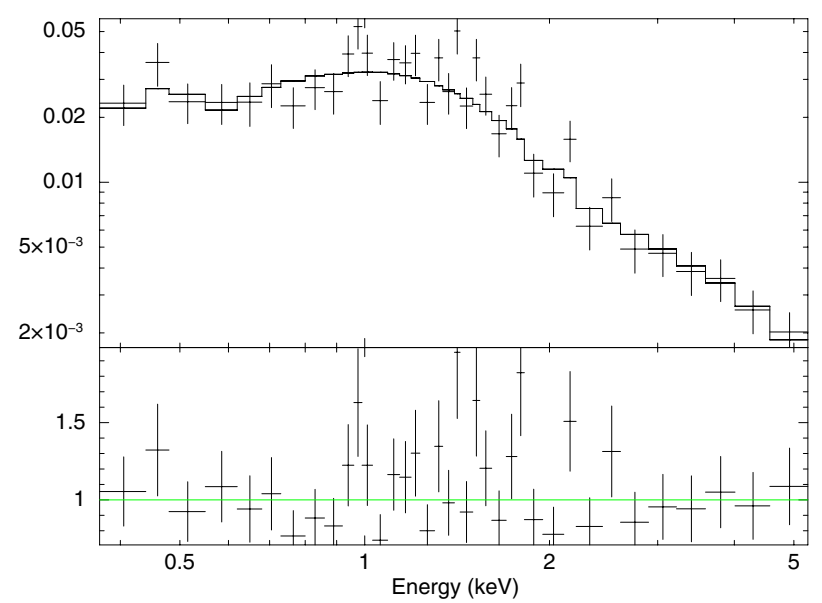

Fig. 3. Powerlaw fit to the Swift X-ray spectrum of RX J2314.9+2243, and fit residuals (lower panel), for the 2007 May 20 observation.

with a radius of $47^{\prime \prime}$, while the background counts were determined in a nearby circle of radius of 235.7".

Spectra were created using the response file swxpc0to12s0_20010101v012.rmf. The data of the second observation were binned with 20 counts per bin and fitted with $\chi^{2}$ statistics. Spectral fits were performed with XSPEC 0.12.7. We used a Galactic foreground column density of $9.55 \times 10^{20} \mathrm{~cm}^{-2}$ which was determined from $E_{B-V}=0.195$ (Schlegel et al. 1998) based on the relation given by Diplas \& Savage (1994). Spectral fits were performed in the observed $0.3-10 \mathrm{keV}$ energy band, unless noted otherwise.

A single powerlaw provides a successful fit to the X-ray spectrum of RX J2314.9+2243 (Fig. 3). The cold absorption, when treated as free parameter, $N_{\mathrm{H}}=8.74 \times 10^{20} \mathrm{~cm}^{-2}$, is larger than the Galactic value listed in Dickey \& Lockman (1990) based on HI data, $N_{\mathrm{HI}}=6.11 \times 10^{20} \mathrm{~cm}^{-2}$, but is actually comparable to the value inferred from the Galactic extinction of $E_{B-V}=0.195, N_{\mathrm{H}, \mathrm{Gal}}=9.55 \times 10^{20} \mathrm{~cm}^{-2}$. We have therefore fixed $N_{\mathrm{H}}=9.55 \times 10^{20} \mathrm{~cm}^{-2}$, and re-run the powerlaw fit. This then gives $\Gamma_{\mathrm{x}}=-2.00 \pm 0.06(0.3-10 \mathrm{keV})$ and $\chi_{\text {red }}^{2}=1.1$. The powerlaw normalization at $1 \mathrm{keV}$ is $5.85 \times 10^{-4} \pm 0.24 \times$ $10^{-4}$ photons $\mathrm{cm}^{-2} \mathrm{~s}^{-1} \mathrm{keV}^{-1}$, implying an unabsorbed flux of $3 \times 10^{-12} \mathrm{erg} \mathrm{cm}^{-2} \mathrm{~s}^{-1}(0.3-10 \mathrm{keV})$.

Based on this best-fit model, we predict a ROSAT PSPC countrate of $0.08 \mathrm{cts} / \mathrm{s}$, similar to the one actually observed with ROSAT, 0.11 cts/s (K06).

In order to check for the presence of an additional "soft excess", as it is commonly found in NLS1 galaxies, we have re-fit the spectrum adding a black body component. We find that the black body is negligible. We also re-fit the data with a single

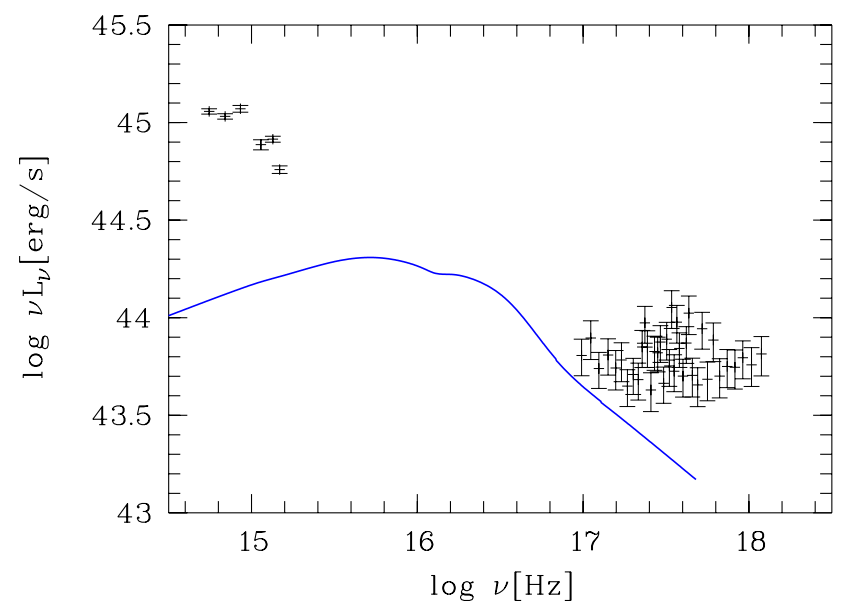

Fig. 4. Swift SED of RX J2314.9+2243 during the 2007 May 20 observation. X-ray data have been corrected for absorption, optical-UV data for Galactic extinction. For comparison, we show the average SED of the (radio-quiet) NLS1 galaxies of Grupe et al. (2010).

powerlaw, excluding all source photons below $2 \mathrm{keV}$. The derived slope, $\Gamma_{\mathrm{x}}=-1.98 \pm 0.3$, is consistent with the $0.3-10 \mathrm{keV}$ value. The Swift SED of RX J2314.9+2243 is shown in Fig. 4, along with the average SED of (radio-quiet) NLS1 galaxies of Grupe et al. (2010; mean values of their Table 6). The comparison shows that the SED of RX J2314.9+2243 deviates significantly from the population of radio-quiet NLS1 galaxies. While its X-ray spectrum is significantly flatter than the population average, its UV spectrum is exceptionally steep.

In order to search for short time variability during the Swift observation, we have produced the X-ray lightcurve of RX J2314.9+2243 with orbit by orbit bins using the online tool at Leicester ${ }^{4}$. The counts are corrected for losses due to bad columns, PSF and vignetting as described in Evans et al. (2009). There is marginal evidence of variability, including a drop in countrate by $\sim 30 \%$ within $6 \mathrm{ks}$ (Fig. 5). Running a $\chi^{2}$ test to judge the significance of variability, we find a probability value of 0.022 for the hypothesis that the light curve is constant.

\section{Radio observations}

RX J2314.9+2243 was detected in the NRAO VLA Sky Survey (NVSS; Condon et al. 1998) at $1.4 \mathrm{GHz}$ with a flux density level of $S_{v, \mathrm{NVSS}}=18.7 \mathrm{mJy}$.

New radio observations of RX J2314.9+2243 were performed with the Effelsberg $100 \mathrm{~m}$ telescope, motivated by its remarkable [OIII] outflow (K06) possibly linked to radio-mode feedback. The observations of RX J2314.9+2243 were carried out on July 07, 2013 within a Fermi-GST related monitoring program of $\gamma$-ray blazars (F-GAMMA program, Fuhrmann et al. 2007, 2014; Angelakis et al. 2008).

The measurements were obtained with the secondary focus heterodyne receivers at 2.64, 4.85, 8.35, 10.45, and $43.00 \mathrm{GHz}$. Since RX J2314.9+2243 is point-like for the Effelsberg beam (e.g., $69^{\prime \prime}$ at $10.8 \mathrm{GHz}$ ) at all frequencies, the observations were performed quasi-simultaneously with cross-scans, that is slewing over the source position, in azimuth and elevation direction with an adaptive number of sub-scans for reaching the desired sensitivity (for details, see also Fuhrmann et al. 2008; Angelakis et al. 2008). Subsequently, pointing off-set correction,

4 http://www.swift.ac.uk/user_objects/ 


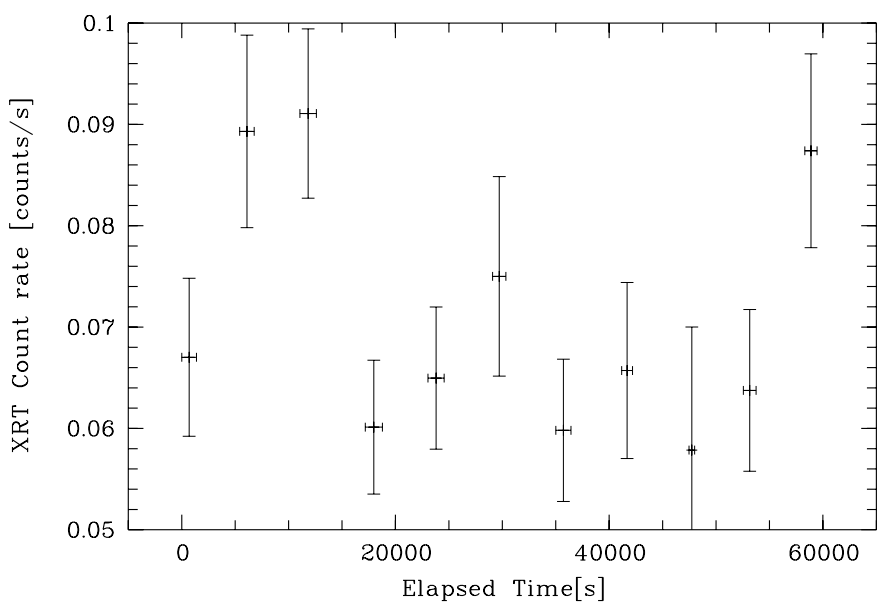

Fig. 5. Swift lightcurve of RX J2314.9+2243 during the 2007 May 20 observation. Data have been corrected for losses due to bad columns, PSF and vignetting.

gain correction, atmospheric opacity correction and sensitivity correction have been applied to the data.

The source was detected at $2.64,4.85$, and $8.35 \mathrm{GHz}$ with flux densities of $12 \pm 2,9 \pm 2$, and $5 \pm 1 \mathrm{mJy}$, respectively. At higher frequencies non-detections imply $3 \sigma$ upper limits of $17 \mathrm{mJy}$ at $10.45 \mathrm{GHz}$ and $56 \mathrm{mJy}$ at $43.00 \mathrm{GHz}$ (Table 4). The radio spectrum obtained from the lower frequency detections is steep with a spectral index of $\alpha_{\mathrm{R}}=-0.76\left(S \propto v^{\alpha}\right)$ between $2.64 \mathrm{GHz}$ and $8.35 \mathrm{GHz}$ (or -0.47 between $2.64 \mathrm{GHz}$ and $4.85 \mathrm{GHz}$ ).

Data were also taken at three other dates in 2013 (Table 1), and flux density measurements are consistent within the errors with the measurements of 2013 July 7 (Table 4). So far, there is no evidence of variability of RX $\mathrm{J} 2314.9+2243$ in the radio band.

\section{Broadband SED}

In order to obtain the broadband multiwavelength SED of RX J2314.9+2243, we have added archival data. RX J2314.9+2243 was observed in the MIR with the Infrared Astronomical Satellite (IRAS) and is detected at 25 and 60 microns (Moshir et al. 1990) with $0.21 \pm 0.02 \mathrm{Jy}$ and $0.36 \pm 0.05 \mathrm{Jy}$, respectively. In the near-UV and far-UV, it was detected with the Galaxy Evolution Explorer (GALEX; Seibert 2012) with $18.42 \pm 0.03 \mathrm{mag}$ and $19.5 \pm 0.1 \mathrm{mag}$, respectively. RX J2314.9+2243 was also mapped with the Wide-field Infrared Survey Explorer (WISE; Wright et al. 2010) and was detected at all four wavebands $(3.4,4.6,12$, and 22 microns) with magnitudes of $10.17 \pm 0.02,9.22 \pm 0.02,6.74 \pm 0.02$, $4.41 \pm 0.03$, respectively. We have obtained the WISE lightcurve of RX J2314.9+2243, and find little evidence of variability. The average WISE fluxes in each band have been used for the SED.

The broadband SED of RX J2314.9+2243 is shown in Fig. $6^{5}$. The optical and UV data have been corrected for Galactic extinction. The SED is characterized by a broad hump from the IR to the UV with a steep decline in the UV. For comparison, we have overplotted the SEDs of two other radioloud NLS1 galaxies, SBS $0846+513$ and $1 \mathrm{H} 0323+342$, and the

\footnotetext{
5 We expect that the emission in most wavebands is dominated by the nucleus, except in the optical band, where the SDSS image reveals faint emission from the host galaxy.
}

single-zone leptonic jet models which Sun et al. (2014) and Yao et al. (2014) obtained fitting the SEDs of these NLS1 galaxies. Their models are based on single-zone synchrotron and inverse Compton (IC) processes to explain the jet emission, with an extra accretion disk contribution when required. The IC emission includes synchrotron self-Compton (SSC) scattering and external Compton (EC) scattering (see Sun et al. 2014, for details). Data points for the two NLS1 galaxies are from D'Ammando et al. (2012) and Yao et al. (2014). Since most of our data are nonsimultaneous, we do not attempt any dedicated SED modeling of RX J2314.9+2243.

\section{A comment on black hole mass and Eddington ratio}

RX J2314.9+2243 harbors a SMBH of mass $M \approx 8 \times$ $10^{7} M_{\odot}$ (K06). This value is at the upper end of typical NLS1 galaxy SMBH masses (e.g., Grupe et al. 2010; Xu et al. 2012), but at the lower end of the blazar SMBH mass distribution (e.g., Cao 2003). This mass estimate is an upper limit, if there is a significant contribution from jet emission to the optical band.

Applying a bolometric correction factor of 10 to the (ROSAT) X-ray luminosity gave $L / L_{\text {edd }}=0.2(\mathrm{~K} 06)$. The same value of $L / L_{\text {edd }}=0.2$ is obtained, if we use Eq. (1) of Grupe et al. (2010) which links the X-ray spectral index with $L / L_{\text {edd }}$ for values of $\Gamma_{\mathrm{x}}>-2.6$. This value of $L / L_{\text {edd }}$ is within the distribution of NLS1 galaxies, but more towards its lower end.

\section{Discussion}

We present our discussion with two possibilities in mind: (1) a scenario, in which the SED of RX J2314.9+2243 is dominated by thermal processes in a dusty LIRG environment; or (2) that its broadband emission is mostly non-thermal in nature and related to a (blazar) jet. Independent of the major power source of the broadband emission, we then discuss the nature of its exceptionally blueshifted [OIII] emission-line component.

\subsection{Emission processes of $R X J 2314.9+2243$}

RX J2314.9+2243 is only mildly radio loud, with radio index $R=10-20$ (where the range of values arises from the use of optical magnitudes from various catalogues; K06). Nevertheless, the tentative transient $\gamma$-ray detection (Berton et al., in prep.) hints at the presence of strong jet activity in this NLS1 galaxy. There are several other indications that parts of its SED are dominated by non-thermal processes. The X-ray spectrum of RX J2314.9+2243 observed with both ROSAT and Swift is unusually flat when compared with the majority of NLS1 galaxies, which, on average, show steep X-ray spectra in the soft X-ray band (e.g., Boller et al. 1996; Grupe 2004; Zhou et al. 2006). Flat-spectrum radio quasars on the other hand, are characterized by flat soft $X$-ray spectra $\left(\Gamma_{\mathrm{x}} \approx-2\right.$ or flatter; e.g., Brinkmann et al. 1997; Giommi et al. 2012; Fan et al. 2012), traced back to non-thermal emission processes, related to jet activity (e.g., Sikora et al. 2013), or an accretion-disk corona.

Furthermore, the UV spectrum of RX J2314.9+2243 is unusually steep for a NLS1 galaxy. One way to explain this, would be intrinsic reddening. At first glance, this is not unusual for luminous infrared galaxies (LIRGs), which tend to occur in gasrich, dusty systems (e.g., Sanders et al. 1988). However, we do 
Table 4. Effelsberg radio measurements.

\begin{tabular}{lcccc}
\hline \hline Frequency $[\mathrm{GHz}]$ & \multicolumn{5}{l}{ Flux density [mJy] } & & \\
& 2013 July 7 & 2013 Feb. 03 & 2013 Feb. 09 & 2013 July 23 \\
\hline 2.64 & $12 \pm 2$ & - & $14 \pm 3$ & - \\
4.85 & $9 \pm 2$ & $7 \pm 1$ & $7 \pm 1$ & $7 \pm 2$ \\
8.35 & $5 \pm 1$ & $5 \pm 1$ & $5 \pm 1$ & $5 \pm 1$ \\
10.45 & $<17$ & - & - & - \\
43.00 & $<56$ & - & - & - \\
\hline
\end{tabular}

Notes. Not all frequencies were observed at all dates. RX J2314.9+2243 was also detected during the NVSS at 1.4 GHz with a flux density of $19 \pm 1 \mathrm{mJy}$.

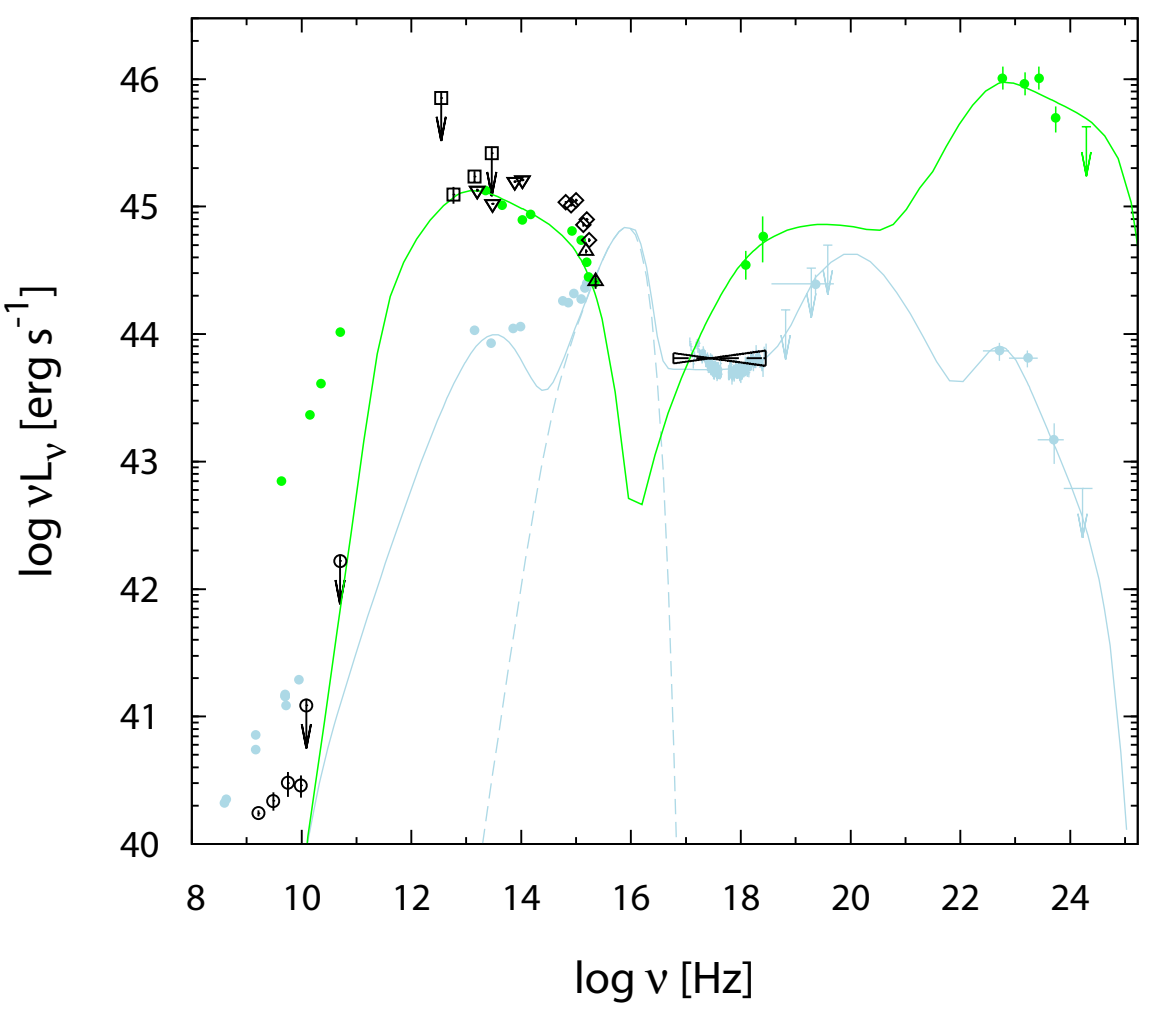

Fig. 6. Broadband SED of RX J2314.9+2243 (black symbols; open circles: NVSS and Effelsberg; open squares: IRAS, open inverted triangles: WISE, open diamonds: Swift UVOT, open triangles: GALEX, black line: Swift X-ray high-state). For comparison, the observed and modeled SEDs of the radio-loud NLS1 galaxies SBS $0846+513$ (green) and $1 \mathrm{H} 0323+342$ (blue) are shown, based on the model fits of Sun et al. (2014) and Yao et al. (2014), respectively. Data for these two galaxies (green and blue circles) are from D'Ammando et al. (2012) and Yao et al. (2014). The solid lines represent the best-fit jet models, while the dashed line represents radiation from the accretion disk (see Sect. 6 for details). (A color version of this figure is available in the online journal.) not find any other strong evidence of significant extinction beyond the Galactic value. The optical Balmer emission lines are consistent with the recombination value (Osterbrock 1989). The Swift observation itself was not deep enough to exclude the possibility that the X-ray spectrum is heavily absorbed and dominated by scattered emission below $10 \mathrm{keV}$ (as is the case in other LIRGs, like NGC 6240). However, in that case, the X-rays should not vary within days, and the intrinsic luminosity would end up above the Eddington luminosity, given that the estimated accretion rate is already close to Eddington.

Therefore, the IR-UV emission of RX J2314.9+2243 is likely dominated by synchrotron emission from a jet. With an SED peaking near $10^{14} \mathrm{GHz}, \mathrm{RX}$ J2314.9+2243 classifies as ISP ("Intermediate Synchrotron Peaked") in the scheme of Abdo et al. (2010). As such, it peaks at higher frequencies than the bulk of the flat-spectrum radio quasars (FSRQs; Giommi et al. 2012) but still within the scatter.

The Effelsberg data imply a steep radio spectrum with a spectral index of -0.76 . This is unlike most blazars with flat spectra, and, at first glance, comes as a surprise, given its possible $\gamma$-ray detection. There are several potential interpretations. First, its (marginally detected) $\gamma$-ray emission could arise from processes other than beamed radiation in a relativistic jet (e.g.,
Ghisellini et al. 2005). The $\gamma$-ray sources detected with Fermi include a few starburst galaxies, and some radio galaxies with misaligned jets (e.g., Abdo et al. 2009c; Ackermann et al. 2012; Grandi 2012; Di Mauro et al. 2014). Second, its radio spectral index could be highly variable, and/or represent the emission in the low-state, where quiescent extended radio structures dominate the radiation. Overall, radio-loud NLS1 galaxies come in two flavors. Those with steep spectral indices $\alpha_{\mathrm{r}}<-0.44$ ( 5 out of 7 with multifrequency measurements in the sample of K06; see also Caccianiga et al. 2014 on a new case), and those with flat spectral indices ( 8 out of 11 with multifrequency measurements in the sample of Yuan et al. 2008). Radio spectra of known beamed individual radio-loud NLS1 galaxies (and classical blazars) can be highly variable (e.g., Angelakis et al. 2014), and change between steep, flat and inverted spectral states depending on epoch of observation and frequency. Long-term radio-monitoring of RX J2314.9+2243 will reveal, whether its spectrum varies strongly, or remains constant; while ongoing $\gamma$-ray observations will be useful to confirm the marginal highenergy detection.

How does the strong, highly shifted [OIII] component in the optical spectrum of RX J2314.9+2243 fit into this picture? Kinematic shifts in the main component of the [OIII] emission 
line (so-called "blue outliers"; e.g., Zamanov et al. 2002; Komossa et al. 2008), and in extra [OIII] emission components ("blue wings"; e.g., Zhang et al. 2011) are present in a fraction of all AGN. Those kinematic shifts, however, are usually much less extreme than that of RX J2314.9+2243. First of all, we note that RX J2314.9+2243 is not a blue outlier. Its [OIII] core component is not blueshifted. Instead, RX J2314.9+2243 shares similarities with a number of radio sources which are characterized by strong [OIII] blue wings, or multipeaked [OIII] profiles (e.g., Morganti et al. 2007; Holt et al. 2008; Nesvadba et al. 2011), traced back in these sources to strong jet-cloud interactions in a dense environment. A similar scenario may apply to RX J2314.9+2243. The particularly high kinematic shift, $v=$ $1260 \mathrm{~km} \mathrm{~s}^{-1}$, of its broad [OIII] component could then be understood in terms of a face-on view, with the jet (and outflow) pointing towards us. Given the low redshift of RX J2314.9+2243, the spatial scale of the outflow could potentially be directly resolved by future high-resolution radio and optical (HST) observations, providing a direct test of the outflow scenario.

\section{Summary and conclusions}

We have presented a multiwavelength study of the radioloud galaxy RX J2314.9+2243, including the first simultaneous optical-UV-X-ray measurements, and the first (and simultaneous) multifrequency radio measurements.

RX J2314.9+2243 shows the triple characteristics of a LIRG, a NLS1 galaxy and a blazar. Different emission processes may dominate at different wavelengths.

Except in X-rays, there is no evidence so far for variability in the IR, optical, UV, and radio band.

Luminous emission in the infrared leads to a LIRG classification. In the majority of these sources, the IR emission arises from re-emission of dust heated by starburst or AGN activity. However, in the case of RX J2314.9+2243, the more likely possibility arises, that its IR-UV emission is instead dominated by synchrotron emission from a jet.

Its steep UV spectrum, $\alpha_{\mathrm{UV}}=1.63$, and flat X-ray spectrum, $\Gamma_{\mathrm{x}}=-2.0$, is unlike the majority of NLS1 galaxies. Since there is no evidence of strong excess extinction, the observed spectral shape is likely intrinsic. This then argues for non-thermal emission processes dominating the SED; synchrotron emission in the IR-UV, an accretion-disk corona in X-rays.

In the radio regime, its spectrum is steep $\left(\alpha_{\mathrm{R}}=-0.76\right)$, more reminiscent of CSS than of blazars. Yet, its occasional $\gamma$-ray detection with Fermi, if confirmed, hints at flaring due to a relativistic jet. The observed radio emission then likely represents emission from a quiescent jet component, and, indeed, the similar radio fluxes observed with NVSS and at a much later epoch with Effelsberg suggest, that RX J2314.9+2243 was not in a flaring state during the latter observation.

Since classical LIRGs are often mergers, we cannot yet exclude the possibility that RX J2314.9+2243 hosts a binary AGN at its center, each contributing differently at different wavelengths. Highest-resolution radio imaging could test this scenario further.

The exceptionally broad and blue-shifted [OIII] emissionline component of RX J2314.9+2243 implies the presence of a strong outflow, plausibly powered by radio-jet feedback, and consistent with a near on-axis viewing angle.

Sources like RX J2314.9+2243 provide us with new constraints on the extremes of AGN activity, and shed new light on the nature of radio-loudness in NLS1 galaxies.
Acknowledgements. We thank Phil Evans, Luigi Foschini, Erlin Qiao, Jing Wang, and Jin Zhang for useful discussions, and Jin Zhang for providing her jet models, and the literature data for SBS $0846+513$, in printable form. We also thank our referee for many useful comments and suggestions. This research is partly based on observations carried out with the following telescopes: (1) the $2.2 \mathrm{~m}$ and $80 \mathrm{~cm}$ telescopes of Xinglong station operated by NAOC; (2) the $100 \mathrm{~m}$ telescope of the MPIfR (Max-Planck-Institut für Radioastronomie) at Effelsberg; and (3) the X-ray observatory Swift. At PSU, Swift is supported by NASA contract NAS5-00136. This research has made use of the NASA/IPAC Extragalactic Database (NED) which is operated by the Jet Propulsion Laboratory, California Institute of Technology, under contract with the National Aeronautics and Space Administration. This work made use of data supplied by the UK Swift Science Data Centre at the University of Leicester. This publication has made use of data products from the Wide-field Infrared Survey Explorer, which is a joint project of the University of California, Los Angeles, and the Jet Propulsion Laboratory/California Institute of Technology, funded by the National Aeronautics and Space Administration. This work was supported by grant NSFC 11273027. I.M. and V.K. are funded by the International Max Planck Research School (IMPRS) for Astronomy and Astrophysics at the Universities of Bonn and Cologne.

\section{References}

Abdo, A. A., Ackermann, M., Ajello, M., et al. 2009a, ApJ, 699, 976 Abdo, A. A., Ackermann, M., Ajello, M., et al. 2009b, ApJ, 707, L142 Abdo, A. A., Ackermann, M., Ajello, M., et al. 2009c, ApJ, 699, 31 Abdo, A. A., Ackermann, M., Agudo, I., et al. 2010, ApJ, 716, 30 Ackermann, M., Ajello, M., Allafort, A., et al. 2012, ApJ, 755, 164 Angelakis, E., Fuhrmann, L., Marchili, N., et al. 2008, Mem. Soc. Astron. It., 79, 1042

Angelakis, E., et al. 2014, A\&A, submitted

Anton, S., Browne, I. W. A., \& Marcha, M. J. 2008, A\&A, 490, 583

Boller, T., Brandt, W. N., \& Fink, H. H. 1996, A\&A, 305, 53

Boroson, T. A., \& Green, R. F. 1992, ApJS, 80, 109

Breeveld, A. A., Curran, P. A., Hoversten, E. A., et al. 2010, MNRAS, 406, 1687

Brinkmann, W., Yuan, W., \& Siebert, J. 1997, A\&A, 319, 413

Burrows, D. N., Hill, J. E., \& Nousek, J. A. 2005, Space Sci. Rev., 120, 165

Caccianiga, A., Antón, S., Ballo, L., et al. 2014, MNRAS, 441, 172

Calderone, G., Foschini, L., Ghisellini, G., et al. 2011, MNRAS, 413, 2365

Cao, X. 2003, ApJ, 599, 147

Capetti, A., \& Balmaverde, B. 2006, A\&A, 453, 27

Cardelli, J. A., Clayton, G. C., \& Mathis, J. S. 1989, ApJ, 345, 245

Condon, J. J., Cotton, W. D., Greisen, E. W., et al. 1998, AJ, 115, 1693

Dallacasa, D., Bondi, M., Alef, W., \& Mantovani, F. 1998, A\&AS, 129, 219

D’Ammando, F., Orienti, M., Finke, J., et al. 2012, MNRAS, 426, 317

D'Ammando, F., Orienti, M., Doi, A., et al. 2013, MNRAS, 433, 952

D'Ammando, F., Larsson, J., Orienti, M., et al. 2014, MNRAS, 438, 3521

Dickey, J. M., \& Lockman, F. J. 1990, ARA\&A, 28, 215

Di Mauro, M., Calore, F., Donato, F., Ajello, M., \& Latronico, L. 2014, ApJ, 780,161

Diplas, A., \& Savage, B. 1994, ApJS, 93, 211

Doi, A., Fujisawa, K., Inoue, M., et al. 2007, PASJ, 59, 703

Doi, A., Asada, K., Nagai, H., et al. 2011, ApJ, 738, 126

Doi, A., Nagira, H., Kawakatu, N., et al. 2012, ApJ, 760, 41

Evans, I. N. 1988, ApJS, 67, 373

Evans, P., Beardmore, A. P., Page, K. L., et al. 2009, MNRAS, 397, 1177 Falcone, A. D., Bond, I. H., Boyle, P. J., et al. 2004, ApJ, 613, 710

Fan, J. H., Yang, J. H., Yuan, Y. H., Wang, J., \& Gao, Y. 2012, ApJ, 761, 125 Foschini, L. 2011, Proc. Science (PoS), NLS1, 24

Foschini, L., Ghisellini, G., Kovalev, Y. Y., et al. 2011, MNRAS, 413, 1671

Foschini, L., Angelakis, E., Fuhrmann, L., et al. 2012, A\&A, 548, A106

Foschini, L., Berton, M., Caccianiga, S., et al. 2014, A\&A, submitted [arXiv: 1409. 3716]

Fuhrmann, L., Zensus, J. A., Krichbaum, T. P., Angelakis, E., \& Readhead, A. C. S. 2007, The First GLAST Symp., AIP Conf. Proc., 921, 249

Fuhrmann, L., Krichbaum, T. P., Witzel, A., et al. 2008, A\&A, 490, 1019

Fuhrmann, L., Angelakis, E., Nestoras, I., et al. 2011, in Proc. Science (PoS), NLS1, 26, published online at http://pos.sissa.it/cgi-bin/reader/ conf. cgi? confid $=126$

Fuhrmann, L., Larsson, S., Chiang, J., et al. 2014, MNRAS, 441, 1899

Ghisellini, G., Tavecchio, F., \& Chiaberge, M. 2005, A\&A, 432, 401

Giommi, P., Polenta, G., Lähteenmäki, A., et al. 2012, A\&A, 541, A160

Giroletti, M., Paragi, Z., Bignall, H., et al. 2011, A\&A, 528, L11

Goodrich, R. W. 1989, ApJ, 340, 190

Grandi, P. 2012, Int. J. Mod. Phys. Conf. Ser., 8, 25

Grupe, D. 2004, AJ, 127, 1799

Grupe, D., Komossa, S., Leighly, K. M., \& Page, K. L. 2010, ApJS, 187, 64 Gu, M., \& Chen, Y., 2010, AJ, 139, 2612 
Hill, J. E., Burrows, D. N., Nousek, J. A., et al. 2004, SPIE, 5165, 217

Holt, J., Tadhunter, C. N., \& Morganti, R. 2008, MNRAS, 387, 639

Kellerman, K. I., Sramek, R., Schmidt, M., Shaffer, D. B., \& Green, R. 1989, AJ, 98, 1195

Komossa, S. 2008, Rev. Mex. Astron. Astrofis., 32, 86

Komossa, S., Voges, W., Xu, D., et al. 2006, AJ, 132, 531

Komossa, S., Xu, D., Zhou, H., et al. 2008, ApJ, 680, 926

Kriss, G. 1994, in Astronomical Data Analysis Software and Systems III, eds.

D. R. Crabtree, R. J. Hanisch, \& J. Barnes, ASP Conf. Ser., 61 (San Francisco: ASP), 437

Leighly, K. 1999, ApJS, 125, 297

Massey, P., \& Strobel, K. 1988, ApJ, 328, 315

Mathur, S. 2000, MNRAS, 314, L17

Morganti, R., Holt, J., Saripalli, L., Oosterloo, T. A., \& Tadhunter, C. N. 2007, A\&A, 476, 735

Moshir, M., et al. 1990, IRAS Faint Source Catalogue, http://ned.ipac. caltech. edu/cgi-bin/ex_refcode?refcode=1990IRASF. C . . DO000M

Nesvadba, N. P. H., Polletta, M., Lehnert, M. D., et al. 2011, MNRAS, 415, 2359

Osterbrock, D. E. 1989, Astrophysics of Gaseous Nebulae and Active Galactic

Nuclei (University Science Books: Mill Valley, CA)

Osterbrock, D. E., \& Pogge, R. 1985, ApJ, 297, 166

Poole, T. S., Breeveld, A. A., Page, M. J., et al. 2008, MNRAS, 383, 627

Roming, P. W. A., Kennedy, T. E., Mason, K., et al. 2005, Space Sci. Rev., 120, 95

Roming, P. W. A., Koch, T. S., Oates, S. R., et al. 2009, ApJ, 690, 163

Sanders, D. B., Soifer, B. T., Elias, J. H., et al. 1988, ApJ, 325, 74
Sani, E., Lutz, D., Risaliti, G., et al. 2010, MNRAS, 403, 1246

Schlegel, D. J., Finkbeiner, D. P., \& Davis, M. 1998, ApJ, 500, 525

Seibert, M. 2012, GALEX all-Sky Catalogue, http://ned.ipac.caltech. edu/cgi-bin/ex_refcode?refcode=2012GASC. .C. . .0000S

Sikora, M., \& Begelman, M. 2013, ApJ, 764, L24

Sikora, M., Janiak, M., Nalewajko, K., et al. 2013, ApJ, 779, 68

Sun, X.-N., Zhang, J., Lin, D.-B., Liang, E.-W., \& Zhang, S.-N. 2014, ApJ, submitted [arXiv: $1406.1934 \mathrm{v} 1]$

Tchekhovskoy, A., Narayan, R., \& McKinney, J. C. 2010, ApJ, 711, 50

Urry, C. M., \& Padovani, P. 1995, PASP, 107, 803

Véron-Cetty, M. P., Véron, P., \& Gonçalves, A. C. 2001, A\&A, 372, 730

Véron-Cetty, M. P., Joly, M., \& Véron, P. 2004, A\&A, 417, 515

Wajima, K., Fujisawa, K., Hayashida, M., et al. 2014, ApJ, 781, 75

Wei, J.-Y., Xu, D., Cao, L., et al. 1998, A\&A, 329, 511

Wilson, A. S., \& Colbert, E. J. M. 1995, ApJ, 438, 62

Wright, E. L. 2006, PASP, 118, 1711

Wright, E. L., Eisenhardt, P. R. M., Mainzer, A. K., et al. 2010, AJ, 140, 1868

Xu, D., Komossa, S., Zhou, H., et al. 2012, AJ, 143, 83

Yao, S., et al. 2014, AJ, submitted

Yuan, W., Zhou, H. Y., Komossa, S., et al. 2008, ApJ, 685, 801

Zamanov, R., Marziani, P., Sulentic, J. W., et al. 2002, ApJ, 576, L9

Zhang, K., Dong, X.-B., Wang, T.-G., et al. 2011, ApJ, 737, 71

Zhou, H.-Y., Wang, T.-G., Dong, X.-B., Zhou, Y.-Y., \& Li, C. 2003, ApJ, 584, 147

Zhou, H., Wang, T., Yuan, W., et al. 2006, ApJS, 166, 128

Zhou, H., Wang, T., Yuan, W., et al. 2007, ApJ, 658, L13 